\title{
Reduced SMAD7 leads to overactivation of TGF-beta signaling in MDS that can be reversed by a specific inhibitor of TGF-beta receptor I kinase
}

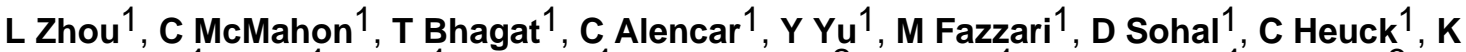

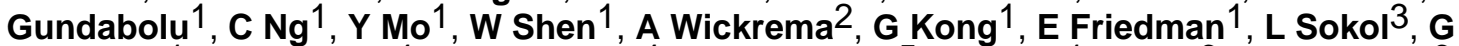 \\ Mantzaris $^{1}$, A Pellagatti ${ }^{4}$, J Boultwood ${ }^{4}$, LC. Platanias ${ }^{5}$, U Steidl ${ }^{1}$, L Yan ${ }^{6}$, JM Yingling ${ }^{6}$,

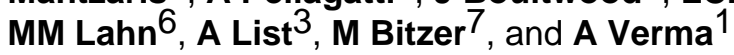 \\ ${ }^{1}$ Albert Einstein College of Medicine, Bronx, NY \\ 2University of Chicago, Chicago, IL \\ ${ }^{3}$ Moffitt Cancer Center, Tampa, FL \\ 4John Radcliffe Hospital, Oxford, UK \\ ${ }^{5}$ Northwestern University Robert H Lurie Cancer Center, Chicago, IL \\ ${ }^{6}$ Eli Lilly \\ ${ }^{7}$ University of Michigan
}

\section{Abstract}

Even though myelodysplastic syndromes are characterized by ineffective hematopoiesis, the molecular alterations that lead to marrow failure have not been well elucidated. We have previously shown that the myelosuppressive TGF- $\beta$ pathway is constitutively activated in MDS progenitors. Since there is conflicting data about upregulation of extracellular TGF-b levels in MDS, we wanted to determine the molecular basis of TGF- $\beta$ pathway overactivation and consequent hematopoietic suppression in this disease. We observed that SMAD7, a negative regulator of TGF- $\beta$ receptor I (TBRI) kinase is markedly decreased in a large meta-analysis of gene expression studies from MDS marrow derived CD34+ cells. SMAD7 protein was also found to be significantly decreased in MDS marrow progenitors when examined immunohistochemically in a bone marrow tissue microarray. Reduced expression of SMAD7 in hematopoietic cells led to increased TGF- $\beta$ mediated gene transcription and enhanced sensitivity to TGF- $\beta$ mediated suppressive effects. The increased TGF- $\beta$ signaling due to SMAD7 reduction could be effectively inhibited by a novel clinically relevant TBRI (ALK5 kinase) inhibitor, LY-2157299. LY-2157299 could inhibit TGF- $\beta$ mediated SMAD2 activation and hematopoietic suppression in primary hematopoietic stem cells. Furthermore, in vivo administration of LY-2157299 ameliorated anemia in a TGF- $\beta$ overexpressing transgenic mouse model of bone marrow failure. Most importantly, treatment with LY-2157199 stimulated hematopoiesis from primary MDS bone marrow specimens. These studies demonstrate that reduction in SMAD7 is a novel molecular alteration in MDS that leads to ineffective hematopoiesis by activating of TGF- $\beta$ signaling in hematopoietic cells. These studies also illustrate the therapeutic potential of TBRI inhibitors in MDS.

Correspondence to: Amit Verma, MD, Chanin 302B, Albert Einstein Cancer Center, 1300 Morris Park Avenue, Bronx, NY 10461, averma@aecom.yu.edu. 


\section{Keywords}

Myelodysplasia; TGF; SMAD7; LY-2157299

\section{INTRODUCTION}

The myelodysplastic syndromes (MDS) are clonal stem cell disorders characterized by cytologic dysplasia and ineffective hematopoiesis(1-3). Although approximately a third of patients may progress to acute leukemia, refractory cytopenias are the principal cause of morbidity and mortality in patients with MDS(4). In fact, approximately two-thirds of patients present with lower risk disease characterized by a hypercellular marrows with increased rates of apoptosis in the progenitor and differentiated cell compartments in the marrow (5-8). Ineffective hematopoiesis arising from abortive maturation leads to peripheral cytopenias. Higher grade or more advanced disease categories are associated with a significant risk of leukemia transformation with a corresponding lower apoptotic index and higher percentage of marrow blasts.

Cytokines play important roles in the regulation of normal hematopoiesis and a balance between the actions of hematopoietic growth factors and myelosuppressive factors is required for optimal production of different hematopoietic cell lineages. Excess production of inhibitory cytokines amplifies ineffective hematopoiesis inherent to the MDS clone. TGF$\beta$ is a myelosuppressive cytokine that has been implicated in the hematopoietic suppression in MDS. The plasma levels of TGF- $\beta$ have been reported to be elevated in some(9-13) but not all studies (14-17) and are supported by greater TGF- $\beta$ immunohistochemical staining in selected studies. In addition to direct myelosuppressive effects, TGF- $\beta$ has also been implicated in the autocrine production of other myelosuppressive cytokines (TNF, IL-6, and IFN $\gamma$ ) in MDS (18). Conflicting data may arise from technical limitations of bone marrow immunohistochemical analyses of a secreted protein as well as the biological heterogeneity of the disease itself. Thus we investigated the role of TGF- $\beta$ in MDS by direct examination of receptor signal activation to conclusively determine its role in the pathogenesis of ineffective hematopoiesis in MDS. We determined that the SMAD2 is upregulated and overactivated in MDS bone marrow progenitors, thereby demonstrating sustained TGF- $\beta$ signal activation in this disease. We also determined that inhibition of SMAD2 activation can stimulate hematopoiesis from primary MDS progenitors, demonstrating its activation by TGF beta receptor as an important event in ineffective hematopoiesis. Since there is conflicting data about upregulation of extracellular TGF- $\beta$ levels in MDS, we next sought to determine the molecular basis of TGF- $\beta$ receptor-I (TBRI) overactivation and subsequent SMAD2 phosphorylation / activation in this disease in the present study. We observed that SMAD7, a negative regulator of TBRI kinase, is markedly decreased in MDS and that this leads overactivation of TGF- $\beta$ signal transduction even in the absence of increased levels of extracellular TGF- $\beta$. Our studies also show that this can be reversed by a clinically relevant novel inhibitor of TGF-- $\beta$ receptor I kinase, LY-2157299, and points to the therapeutic potency of this approach in MDS.

\section{MATERIALS AND METHODS}

\section{Cells Lines and Reagents}

Human CD34+ cells were isolated from bone marrows of normal or MDS patients, after obtaining informed consent approved by the institutional review board of Albert Einstein College of Medicine. Bone marrow CD34+ cells from various normal donors were also obtained from AllCells (Emeryville, CA). K562 and HS-5 cell lines were purchased from ATCC. Erythropoietin and Transforming growth factor- $\beta 1$ (TGF- $\beta 1$ ) were bought from 
R\&D Systems (Minneapolis, MN). The phos-SMAD2 (S465/467) and actin antibodies were from Cell Signaling Technology (Beverly, MA), and SMAD2 antibody from Invitrogen. TBRI inhibitor LY-2157299 was provided by Eli Lilly. LY-2157299 was diluted in DMSO (20 $\mathrm{mM}$ stock solution) and kept at $-20^{\circ} \mathrm{C}$ until use.

\section{Cell Lysis and immunoblotting}

Cells were lysed in phosphorylation lysis buffer as previously described (19). In the experiments in which the effects of LY-2157299 were studied, DMSO (diluent)-treated cells were used as control. Immunoblotting was performed as previously described (19).

\section{ShRNA knockdown experiments: (only use nucleofection, not virus)}

The Human Lentiviral shRNAmir (pGIPZ) containing the hairpin sequence (TGCTGTTGACAGTGAGCGAGCTGTGTTGCTGTGAATCTTATAGTGAAGCCACAG ATGTATAA GATTCACAGCAACACAGCCTGCCTACTGCCTCGGA) targeting SMAD7 and the non-silencing negative control were obtained from OpenBiosystems (OpenBiosystems, AL). Nucleofection of CD34+ cells or MDS BM MNCs was performed according to the manufacturer's instructions, by using the Nucleofector machine (Amaxa, Germany). The $\mathrm{CD} 34^{+}$cells were thawed and cultured for 2 hours, $2 \times 10^{6}$ cells were resuspended in $100 \mu \mathrm{l}$ human $\mathrm{CD} 34^{+}$nucleofection solution (Amaxa, Germany). Samples were transferred into cuvettes and transfected by using program U08. CD $34^{+}$cells were collected, dispensed in the wells of a 24-well plate containing $1 \mathrm{ml}$ prewarmed StemSpan (StemCell Tech), supplemented with $100 \mathrm{ng} / \mathrm{ml}$ human FLt-3, SCF and TPO. The lentiviral transfected $\mathrm{CD} 34^{+}$cells were sorted 24 hours later according to the GFP intensity using Moflow (Becton Dickinson, CA). Quantitative RT-PCR for SMAD7 expression was done on total RNA from HS-5 and K562 cells obtained by RNAeasy mini kit (Qiagen, CA). cDNA was synthesized with SuperScript Reverse Transcriptase III (Invitrogen). Real-time PCR was performed with SYBR green PCR Master Mix (Applied Biosystems, Foster City, CA) and SMAD7 and GAPDH (control) were simultaneously amplified with specific primers.

Primers for GADPH: Forward: 5'-CGACCACTTTGTCAAGCTCA-3' Reverse: 5'CCCTGTTGCTGTAGCCAAAAT-3'

Primers for SMAD7: Forward:5'-GACAGCTCAATTCGGACAAC-3', Reverse: 5' TCTCGTAGTCGAAAGCCTTG-3'

\section{Meta-analysis}

NCBI's GEO database was searched for gene expression studies on MDS and normal CD34 cells. A total of 3 MDS datasets with 89 unique MDS CD34+ studies $(20,21)($ ref BJH) and 61 normal CD34+ datasets from 6 studies (20-24) were identified and downloaded. The data was integrated using unigene IDs. Studies were done using Affymetrix U133A/B and Plus 2.0 platforms. After interarray quantile normalization, SMAD7 expression was assessed and visually represented as a heat map using R statistical software. All genes belonging to TGF$\mathrm{b}$ signaling pathway were examined in the database. To strengthen the criteria for selecting genes that were differentially expressed in MDS, we only selected genes that were significantly different in the combined dataset as well as in the 2 major MDS cohorts $(20,25)$ when studied independently.

\section{Murine Experiments}

Transgenic mice expressing a fusion gene (Alb/TGF) consisting of modified porcine TGF$\beta 1 \mathrm{cDNA}$ under the control of the regulatory elements of the mouse albumin gene (26) were used under animal institute approved protocol. Mice were given LY-2157299 at a dose of 
$100 \mathrm{mg} / \mathrm{kg} /$ day in $\mathrm{NaCMC} / \mathrm{SLS} / \mathrm{PVP} /$ antifoam solution by gastric lavage using a curved $14 \mathrm{G}$ needle. Blood counts were analyzed by Advia machine. Mice femurs were flushed and bone marrows cells were used for clonogenic assays.

TGF- $\beta$ gene reporter assay-HS- 5 cells were plated in six-well plates 24 hours before transfection. Cells were transfected in triplicate with SuperFect (Qiagen, CA) according to the manufacturer's instruction using the reporter plasmid (pGL2-3TP-Lux)(27). pSV-B-Gal was used to normalize the transfection efficiency. After an overnight incubation, the medium was replaced with serum-free medium with or without LY-215as necessary for the experiment. After 48 hours, cells were treated with $10 \mathrm{ng} / \mathrm{ml}$ TGF $\beta 1$. Cells were harvested 24 hours later in reporter lysis buffer (Promega, WI), and luciferase and $\beta$-galactosidase activities were determined using Dual Luciferase System (Promega, WI).

Hematopoietic progenitor cell assays-Hematopoietic progenitor colony formation was determined by clonogenic assays in methylcellulose, as in our previous studies $(19,28)$. All participants in the study signed informed consent, approved by the institutional review board of Albert Einstein College of Medicine. Granulocyte/macrophage colony-forming (CFU-GM) units and erythroid burst forming units (BFU-E) from bone marrow samples were scored on day 14 of culture.

\section{Immunohistochemistry on Bone Marrow Tissue Microarray}

Tissue microarrays (TMAs) were constructed from formalin-fixed, paraffin-embedded bone marrow core biopsies from patients with MDS and control patients with anemia whose bone marrow showed no evidence of neoplasia. The tissue blocks were procured from Jacobi Hospital after approval by the Internal Review Board. For each patient, three $0.5 \mathrm{~mm}$ cores were placed in a tissue array using a manual arrayer (Chemicon International, Tenecula, CA). Sections of the TMAs were cut to $5 \mu \mathrm{m}$ thickness, placed on positively charged slides, and heated to $60^{\circ} \mathrm{C}$ for one hour. They were then deparaffinized in xylene and rehydrated with graded alcohols. Endogenous peroxidase activity was quenched with 3\% hydrogen peroxide. Antigen retrieval was accomplished by microwaving the slides in Dako Target Retrieval Solution, pH 6.0 (Dako Cytomation, Dako, Carpinteria, CA, USA), and subseqently steaming them in a vegetable steamer for 30 minutes. The slides were stained using a rabbit polyclonal anti-SMAD7 antibody, provided at 1:200 dilution, followed by Dako EnVision labeled polymer-HRP anti-rabbit antibody. Antibody binding was detected using DAB chromogen (Cell Marque, Rocklin, CA, USA). The slides were lightly counterstained with hematoxylin, dehydrated with graded alcohols, cleared with xylene, and coverslipped using Cytoseal 60 (Thermo Scientific, Waltham, MA, USA). The tissue cores were then scored for weak versus strong staining for SMAD7 by a hematopathologist who was blinded to the patient identities. Tissue cores that did not contain at least $10 \%$ evaluable marrow were excluded from the analysis.

\section{RESULTS}

\section{SMAD7 is reduced in bone marrow progenitors in low grade MDS}

To determine the molecular events that lead to overactivation of TGF- $\beta$ signaling in MDS, we examined the expression of genes that are involved in and regulate the TGF- $\beta$ signaling cascade in a large number of MDS patient samples. We used a meta-analysis of publicly available microarray studies in MDS, comprising a total of 89 unique MDS patient CD34+ gene expression profiles and 61 normal CD34+ controls. These data were obtained from published studies (20-25,29), were integrated using Unigene protein IDs, subjected to interarray normalization and then used for analysis. This strategy was shown to be biologically valid strategy for analysis previously by us (30). Our analysis of this integrated 
dataset demonstrated that the SMAD7, a negative regulator of the TGF- $\beta$ receptor kinase, was the most significantly differentially expressed gene in MDS and was markedly reduced in most cases (Fig 1, mean $\log 2$ expression 8.31 in controls vs 6.32 in mds cases, p <0.0001, Bejmanin Hochberg correction, multiple testing). Subgroup analysis revealed that SMAD7 was significantly reduced ( $>2$ mean log fold reduction) in both low and high grade MDS samples, though most of the cases examined belonged to low risk MDS (83/89 cases). Subgroup analysis within low grade MDS revealed lower expression in cases with and without deletion of chromosome 5q, demonstrating that decrease in SMAD7 is an widespread and significant change in MDS bone marrow stem cells.

To confirm these results, bone marrows of patients with MDS were assessed for the SMAD7 expression by immunohistochemistry. A tissue microarray containing bone marrow samples from MDS and control bone marrow biopsies was prepared to minimize staining variability between individual slides. MDS bone marrow samples were compared to 24 age matched controls with non-MDS causes of cytopenias [iron deficiency anemia, chemotherapy related anemia, hyperplenism, drug induced marrow suppression, autoimmune anemia,

Myelofibrosis and unexplained cytopenias in the absence of any dysplasia]. Significant reduction in SMAD7 was seen in bone marrow cells of patients with low risk MDS (75\% of samples had reduced expression vs $9 \%$ of controls, $\mathrm{P}<0.05$, proportions test), (Fig 2). Since SMAD7 is ubiquitously expressed, we also investigated the phenotypes of bone marrow cells that had reduced levels of this protein. Histological examination revealed that SMAD7 was reduced in hematopoietic progenitors of all lineages in MDS, including erythroid and myeloid progenitors.

\section{Reduction in SMAD7 can lead to increased sensitivity to suppressive effects of TGF- $\beta$ on hematopoiesis}

We wanted to study the effect of SMAD7 reduction on TGF- $\beta$ signaling in the hematopoietic system. A lentiviral vector based shRNA vector targeting SMAD7 was developed (Fig 3A) and was successfully able to inhibit SMAD7 expression when compared to scrambled control in various hematopoietic cells. (Fig 3B). Primary bone marrow derived CD34+ stem cells were infected with lentiviruses containing anti-SMAD7 and control shRNA constructs and then GFP selected and grown in methylcellulose in the presence and absence of varying amounts of TGF- $\beta$. Downregulation of SMAD7 led to significantly increased sensitivity to TGF- $\beta$ and very low amounts of TGF- $\beta$ was able to significantly inhibit erythroid colony formation when compared to controls, .

Next, we wanted to study the effect of SMAD7 downregulation on TGF- $\beta$ signaling at the transcriptional level. Luciferase reporter assays that were dependant on SMAD2 binding demonstrated that shRNA mediated downregulation of SMAD7 led to increase in TGF- $\beta$ mediated gene transcription in bone marrow stromal cells stably transfected with lentiviral shRNAs, Since previous studies have shown that SMAD7 can inhibit TGF- $\beta$ receptor I kinase (TBRI) in other model systems, we hypothesized that reduction in SMAD7 levels leads to overactivation of TBRI and subsequent downstream signaling in hematopoietic cells in MDS. To test this we used a specific inhibitor of TBRI kinase (LY-2157299) and observed that inhibition of this kinase abrogates the stimulation of TGF- $\beta$ gene transcription that is induced by SMAD7 downregulation.

\section{LY-2157299 is an effective and functionally active inhibitor of TGF- $\beta$ signaling in hematopoietic cells}

Having demonstrated an important role of SMAD7 reduction and subsequent TGF- $\beta$ receptor I kinase activation in hematopoiesis, we next wanted to test the efficacy of LY-2157299, a novel clinically relevant small molecule inhibitor of TBRI (31-35). We first 
demonstrated that LY-2157299 was able to potently inhibit TBRI mediated SMAD2 activation in hematopoietic cells (Fig 4A,B). Next, we tested whether LY-215 was able to reverse the inhibitory effects of TGF- $\beta$ in primary hematopoietic stem cells. Primary CD34+cells were grown in methylcellulose in the presence and absence of TGF- $\beta$ and LY-2157299. As predicted, TGF- $\beta$ led to quantitative and qualitative inhibition of hematopoietic colonies (Fig 4C,D). LY-215 was also able to abrogate the suppressive effects of TGF- $\beta$ on erythroid and myeloid colonies in a dose dependant manner (Fig 4C,D) thus demonstrating the its functional ability in hematopoiesis.

\section{LY-2157299 can improve anemia in a model of bone marrow failure}

To further examine the efficacy of LY-2157299 in vivo, tested it in a transgenic mouse expressing a fusion gene (Alb/TGF) consisting of modified porcine TGF- $\beta$ cDNA under the control of the regulatory elements of the mouse albumin gene. (26). We have shown that these mice constitutively secrete TGF- $\beta$, become anemic and have histologic marrow findings that mimic human MDS, thus serving as an in vivo model of bone marrow failure. (36) These mice were randomized into treatment or placebo groups on the basis of pretreatment hemocrits. Blood counts were measured after 14 days of oral administration of LY-2157299 or vehicle. TBRI inhibitor treatment led to inhibition of constitutive SMAD2 activation in murine bone marrows (Fig 5) and significant increases in hematocrit in these mice demonstrating the specificity of LY-2157299 in inhibiting TGF- $\beta$ signaling and abrogating the hematopoietic defects induced by it. (Fig 5)

\section{LY-2157299 can stimulate MDS hematopoiesis}

Finally, we tested the ability of LY-2157299 in vitro in 10 primary MDS bone marrow samples. These patients had low grade MDS and did not have increased blast counts (Table 1). Mononuclear cells from the bone marrow were grown in methylcellulose with cytokines in the presence and absence of varying doses of LY-2157299. MDS bone marrow cells exhibit poor hematopoietic colony formation in concordance with the hematopoietic failure seen in these patients (Table 1). Treatment with the TBRI inhibitor, LY-2157299, resulted in a significant increase in Erythroid (BFU-E) and Myeloid (CFU-Granulocytic Monocytic) colony numbers in all patients (Results depicted as means Fig 6), These results point to a high therapeutic potential of TBRI inhibition by LY-2157299 in low grade MDS.

\section{DISCUSSION}

The discovery of effective treatments for MDS has been challenged by the limited insight into molecular pathogenesis of the ineffective hematopoiesis seen in these disorders. Even though TGF- $\beta$ levels have been shown to be elevated in subset of MDS cases, our previous work has shown that intracellular signaling is activated in large proportion of cases. Thus, based on our findings, SMAD7 downregulation may be the key mechanism driving the suppression of hematopoiesis in MDS via overactivation of the TGF-SMAD2 pathway. This would also explain increased TGF- $\beta$ signal transduction even in the absence of large amounts of circulating TGF- $\beta$ that has been observed in various studies in MDS patients (14-17).

SMAD7 is an important regulatory protein that has been implicated in TGF mediated effects in many disease and cancer models (37). In fact genome wide association studies have shown SMAD7 snps to be most significant alterations (38) in colorectal cancer demonstrating its importance in maintaining cellular homoeostasis. Another recent study showed that decreased SMAD7 is also seen in idiopathic pulmonary fibrosis and contributes to TGF- $\beta$ mediated fibrosis seen in this disease (39). Our studies demonstrate the role of SMAD7 in hematologic malignancies for the first time and implicate its reduction as an 
important molecular alteration in preleukemic bone marrow failure disorders such as MDS. The bone marrow at earlier stages of MDS is hypercellular and is characterized by ineffective hematopoiesis affecting both stem and progenitor cells. Since TGF- $\beta$ is a well known critical regulator of hematopoiesis and affects both stem and progenitor growth and maturation (40), increased TGF- $\beta$ signaling may potentially explain the dysplastic appearance of progenitors as well as ineffective hematopoiesis of stem cells seen in these patients

There could be many possible reasons for SMAD7 reduction in MDS. Nearly $40 \%$ of MDS cases are characterized by deletions affecting chromosomes 5, 7, 18, 20, Y and others. In fact, the deletion of chromosome 18/18q, where the SMAD7 gene is located, was noticed to be the 4th commonest deletion in the largest cohort of MDS patients described recently (41). Additionally, it has been shown that reduction in levels of genes that are deleted in MDS can have functional consequences on disease pathobiology as illustrated recently by the deletion of ribosome gene, RPS14, in subset of MDS with chr5q deletion (42). Thus, SMAD7 reduction could be a result of $18 \mathrm{q}$ deletions seen in patients. Recent studies using high resolution array based technologies have shown that small deletions can also be seen commonly in MDS and thus can also affect the SMAD7 locus in patients that do not have large 18q deletions as seen on conventional karyotyping studies (43). In addition to genomic deletions, gene silencing in MDS also occurs via aberrant promoter methylation. $(44,45)$. In fact, DNA methyltransferase inhibitors, 5-azacytidine and decitabine, have been approved for the treatment of this disease. Furthermore, newer data from genome wide methylation studies suggests that epigenetic silencing may be a dominant pathological alteration in the disease pathophysiology of MDS (46). Thus, it is quite possible SMAD7 is also silenced epigenetically in MDS and this may result in increased TGF beta signaling and resulting hematopoietic suppression.

Most importantly, our results demonstrate that the activation of TGF- $\beta$ pathway by SMAD7 reduction can be reversed in TBRI inhibition, especially with a clinically relevant inhibitor that is effective in vitro and in vivo. Ineffective hematopoiesis is the cause of most of morbidity in patients with low risk MDS. Two thirds of all MDS cases are at the low risk stage, have a lower chance of progressing to leukemia and mainly suffer problems associated with low blood counts. Thus strategies aimed at raising blood counts are needed to treat these patients. Our data provides a rationale for using inhibitors of TGF- $\beta$ signaling and especially inhibitors of TBRI in this stage of MDS. The type I TGF- $\beta$ receptor selectively participates in TGF- $\beta$ signaling while other activin like and TGF-beta receptors can also participate in BMP and activin ligand signaling (47,48). Therefore, LY-2157299 selectively inhibits TGF- $\beta$ signaling, as it has demonstrated specificity for the TGF- $\beta$ type I receptor. LY-2157299 is a selective dihydropyrrolopyrazole, small molecule inhibitor that competitively binds to the ATP binding site of the TBRI kinase domain. (31-34) This compound is clinically relevant and is presently being tested in Phase I trials in advanced malignancies. Additionally other small molecule and large molecule TBRI inhibitors are also in development $(33,49)$ for various other indications. Our findings provide a preclinical rationale for bringing LY-2157299 and other similar agents into clinical trials for MDS.

\section{Acknowledgments}

Supported by NIH 1R01HL082946. Partnership for cures grant, ACS RSG-09-037 and Leukemia and Lymphoma society Translational research award to AV, NIH RO1 AG029138 to LCP and Immunology and Immunooncology Training Program T32 CA009173 to LZ

\section{References}

1. Heaney ML, Golde DW. Myelodysplasia. N Engl J Med 1999;340:1649-60. [PubMed: 10341278] 
2. Sanz GF, Sanz MA, Greenberg PL. Prognostic factors and scoring systems in myelodysplastic syndromes. Haematologica 1998;83:358-68. [PubMed: 9592987]

3. Greenberg PL. Biologic nature of the myelodysplastic syndromes. Acta Haematol 1987;78(Suppl 1): 94-9. [PubMed: 2829490]

4. Greenberg P, Cox C, LeBeau MM, et al. International scoring system for evaluating prognosis in myelodysplastic syndromes. Blood 1997;89:2079-88. [PubMed: 9058730]

5. Raza A, Gezer S, Mundle S, et al. Apoptosis in bone marrow biopsy samples involving stromal and hematopoietic cells in 50 patients with myelodysplastic syndromes. Blood 1995;86:268-76. [PubMed: 7795232]

6. Greenberg PL. Apoptosis and its role in the myelodysplastic syndromes: implications for disease natural history and treatment. Leuk Res 1998;22:1123-36. [PubMed: 9922076]

7. Westwood NB, Mufti GJ. Apoptosis in the myelodysplastic syndromes. Curr Hematol Rep 2003;2:186-92. [PubMed: 12901339]

8. Ohshima K, Karube K, Shimazaki K, et al. Imbalance between apoptosis and telomerase activity in myelodysplastic syndromes: possible role in ineffective hemopoiesis. Leuk Lymphoma 2003;44:1339-46. [PubMed: 12952227]

9. Zorat F, Shetty V, Dutt D, et al. The clinical and biological effects of thalidomide in patients with myelodysplastic syndromes. Br J Haematol 2001;115:881-94. [PubMed: 11843822]

10. Allampallam K, Shetty V, Mundle S, et al. Biological significance of proliferation, apoptosis, cytokines, and monocyte/macrophage cells in bone marrow biopsies of 145 patients with myelodysplastic syndrome. Int J Hematol 2002;75:289-97. [PubMed: 11999358]

11. Allampallam K, Shetty V, Hussaini S, et al. Measurement of mRNA expression for a variety of cytokines and its receptors in bone marrows of patients with myelodysplastic syndromes. Anticancer Res 1999;19:5323-8. [PubMed: 10697556]

12. Powers MP, Nishino H, Luo Y, et al. Polymorphisms in TGFbeta and TNFalpha are associated with the myelodysplastic syndrome phenotype. Arch Pathol Lab Med 2007;131:1789-93. [PubMed: 18081437]

13. Akiyama T, Matsunaga T, Terui T, et al. Involvement of transforming growth factor-beta and thrombopoietin in the pathogenesis of myelodysplastic syndrome with myelofibrosis. Leukemia 2005;19:1558-66. [PubMed: 16034467]

14. Taketazu F, Miyagawa K, Ichijo H, et al. Decreased level of transforming growth factor-beta in blood lymphocytes of patients with aplastic anemia. Growth Factors 1992;6:85-90. [PubMed: 1591018]

15. Gyulai Z, Balog A, Borbenyi Z, Mandi Y. Genetic polymorphisms in patients with myelodysplastic syndrome. Acta Microbiol Immunol Hung 2005;52:463-75. [PubMed: 16400883]

16. Aguayo A, Kantarjian H, Manshouri T, et al. Angiogenesis in acute and chronic leukemias and myelodysplastic syndromes. Blood 2000;96:2240-5. [PubMed: 10979972]

17. Yoon SY, Li CY, Lloyd RV, Tefferi A. Bone marrow histochemical studies of fibrogenic cytokines and their receptors in myelodysplastic syndrome with myelofibrosis and related disorders. Int J Hematol 2000;72:337-42. [PubMed: 11185990]

18. Verma A, List AF. Cytokine targets in the treatment of myelodysplastic syndromes. Curr Hematol Rep 2005;4:429-35. [PubMed: 16232378]

19. Verma A, Deb DK, Sassano A, et al. Cutting edge: activation of the p38 mitogen-activated protein kinase signaling pathway mediates cytokine-induced hemopoietic suppression in aplastic anemia. $\mathrm{J}$ Immunol 2002;168:5984-8. [PubMed: 12055203]

20. Pellagatti A, Cazzola M, Giagounidis AA, et al. Gene expression profiles of CD34+ cells in myelodysplastic syndromes: involvement of interferon-stimulated genes and correlation to FAB subtype and karyotype. Blood 2006;108:337-45. [PubMed: 16527891]

21. Sternberg A, Killick S, Littlewood T, et al. Evidence for reduced B-cell progenitors in early (lowrisk) myelodysplastic syndrome. Blood 2005;106:2982-91. [PubMed: 16076868]

22. Su AI, Wiltshire T, Batalov S, et al. A gene atlas of the mouse and human protein-encoding transcriptomes. Proc Natl Acad Sci U S A 2004;101:6062-7. [PubMed: 15075390]

23. Oswald J, Steudel C, Salchert K, et al. Gene-expression profiling of CD34+ hematopoietic cells expanded in a collagen I matrix. Stem Cells 2006;24:494-500. [PubMed: 16166251] 
24. Eckfeldt CE, Mendenhall EM, Flynn CM, et al. Functional analysis of human hematopoietic stem cell gene expression using zebrafish. PLoS Biol 2005;3:e254. [PubMed: 16089502]

25. Boultwood J, Pellagatti A, Cattan H, et al. Gene expression profiling of CD34+ cells in patients with the 5q- syndrome. Br J Haematol 2007;139:578-89. [PubMed: 17916100]

26. Sanderson N, Factor V, Nagy P, et al. Hepatic expression of mature transforming growth factor beta 1 in transgenic mice results in multiple tissue lesions. Proc Natl Acad Sci U S A 1995;92:2572-6. [PubMed: 7708687]

27. Yu L, Hebert MC, Zhang YE. TGF-beta receptor-activated p38 MAP kinase mediates Smadindependent TGF-beta responses. Embo J 2002;21:3749-59. [PubMed: 12110587]

28. Verma A, Deb DK, Sassano A, et al. Activation of the p38 mitogen-activated protein kinase mediates the suppressive effects of type I interferons and transforming growth factor-beta on normal hematopoiesis. J Biol Chem 2002;277:7726-35. [PubMed: 11773065]

29. Bhatia M. Molecular Signatures Orchestrating fate of Human Hematopoietic Stem Cells Originating From Different Stages of Ontogeny. Accession No. GSE3823.

30. Sohal D, Yeatts A, Ye K, et al. Meta-analysis of microarray studies reveals a novel hematopoietic progenitor cell signature and demonstrates feasibility of inter-platform data integration. PLoS ONE 2008;3:e2965. [PubMed: 18698424]

31. Liu Z, Kobayashi K, van Dinther M, et al. VEGF and inhibitors of TGFbeta type-I receptor kinase synergistically promote blood-vessel formation by inducing alpha5-integrin expression. J Cell Sci 2009;122:3294-302. [PubMed: 19706683]

32. Li HY, Wang Y, Heap CR, et al. Dihydropyrrolopyrazole transforming growth factor-beta type I receptor kinase domain inhibitors: a novel benzimidazole series with selectivity versus transforming growth factor-beta type II receptor kinase and mixed lineage kinase-7. J Med Chem 2006;49:2138-42. [PubMed: 16539403]

33. Yingling JM, Blanchard KL, Sawyer JS. Development of TGF-beta signalling inhibitors for cancer therapy. Nat Rev Drug Discov 2004;3:1011-22. [PubMed: 15573100]

34. Bueno L, de Alwis DP, Pitou C, et al. Semi-mechanistic modelling of the tumour growth inhibitory effects of LY2157299, a new type I receptor TGF-beta kinase antagonist, in mice. Eur J Cancer 2008;44:142-50. [PubMed: 18039567]

35. Calvo-Aller E, B J, Glatt S, Cleverly A, Lahn M, Arteaga L, Rothenberg ML, Carducci MA. First human dose escalation study in patients with metastatic malignancies to determine safety and pharmacokinetics of LY2157299, a small molecule inhibitor of the transforming growth factorbeta receptor I kinase. J Clin Oncol 2008;26 May 20 suppl; abstr 14554) 2008.

36. Zhou L, Nguyen AN, Sohal D, et al. Inhibition of the TGF-beta receptor I kinase promotes hematopoiesis in MDS. Blood 2008;112:3434-43. [PubMed: 18474728]

37. Akhurst RJ. TGF beta signaling in health and disease. Nat Genet 2004;36:790-2. [PubMed: 15284845]

38. Tenesa A, Farrington SM, Prendergast JG, et al. Genome-wide association scan identifies a colorectal cancer susceptibility locus on 11q23 and replicates risk loci at 8q24 and 18q21. Nat Genet 2008;40:631-7. [PubMed: 18372901]

39. Liu G, Friggeri A, Yang Y, et al. miR-21 mediates fibrogenic activation of pulmonary fibroblasts and lung fibrosis. J Exp Med 2010;207:1589-97. [PubMed: 20643828]

40. He W, Dorn DC, Erdjument-Bromage H, Tempst P, Moore MA, Massague J. Hematopoiesis controlled by distinct TIF1gamma and Smad4 branches of the TGFbeta pathway. Cell 2006;125:929-41. [PubMed: 16751102]

41. Haase D, Germing U, Schanz J, et al. New insights into the prognostic impact of the karyotype in MDS and correlation with subtypes: evidence from a core dataset of 2124 patients. Blood 2007;110:4385-95. [PubMed: 17726160]

42. Ebert BL, Pretz J, Bosco J, et al. Identification of RPS14 as a 5q- syndrome gene by RNA interference screen. Nature 2008;451:335-9. [PubMed: 18202658]

43. Gondek LP, Tiu R, O’Keefe CL, Sekeres MA, Theil KS, Maciejewski JP. Chromosomal lesions and uniparental disomy detected by SNP arrays in MDS, MDS/MPD, and MDS-derived AML. Blood 2008;111:1534-42. [PubMed: 17954704] 
44. Kroeger H, Jelinek J, Estecio MR, et al. Aberrant CpG island methylation in acute myeloid leukemia is accentuated at relapse. Blood 2008;112:1366-73. [PubMed: 18523155]

45. Figueroa ME, Skrabanek L, Li Y, et al. MDS and secondary AML display unique patterns and abundance of aberrant DNA methylation. Blood. 2009

46. Jiang Y, Dunbar A, Gondek LP, et al. Aberrant DNA methylation is a dominant mechanism in MDS progression to AML. Blood 2009;113:1315-25. [PubMed: 18832655]

47. Massague J, Seoane J, Wotton D. Smad transcription factors. Genes \& development 2005;19:2783-810. [PubMed: 16322555]

48. Shi Y, Massague J. Mechanisms of TGF-beta signaling from cell membrane to the nucleus. Cell 2003;113:685-700. [PubMed: 12809600]

49. Akhurst RJ. Large- and small-molecule inhibitors of transforming growth factor-beta signaling. Curr Opin Investig Drugs 2006;7:513-21. 

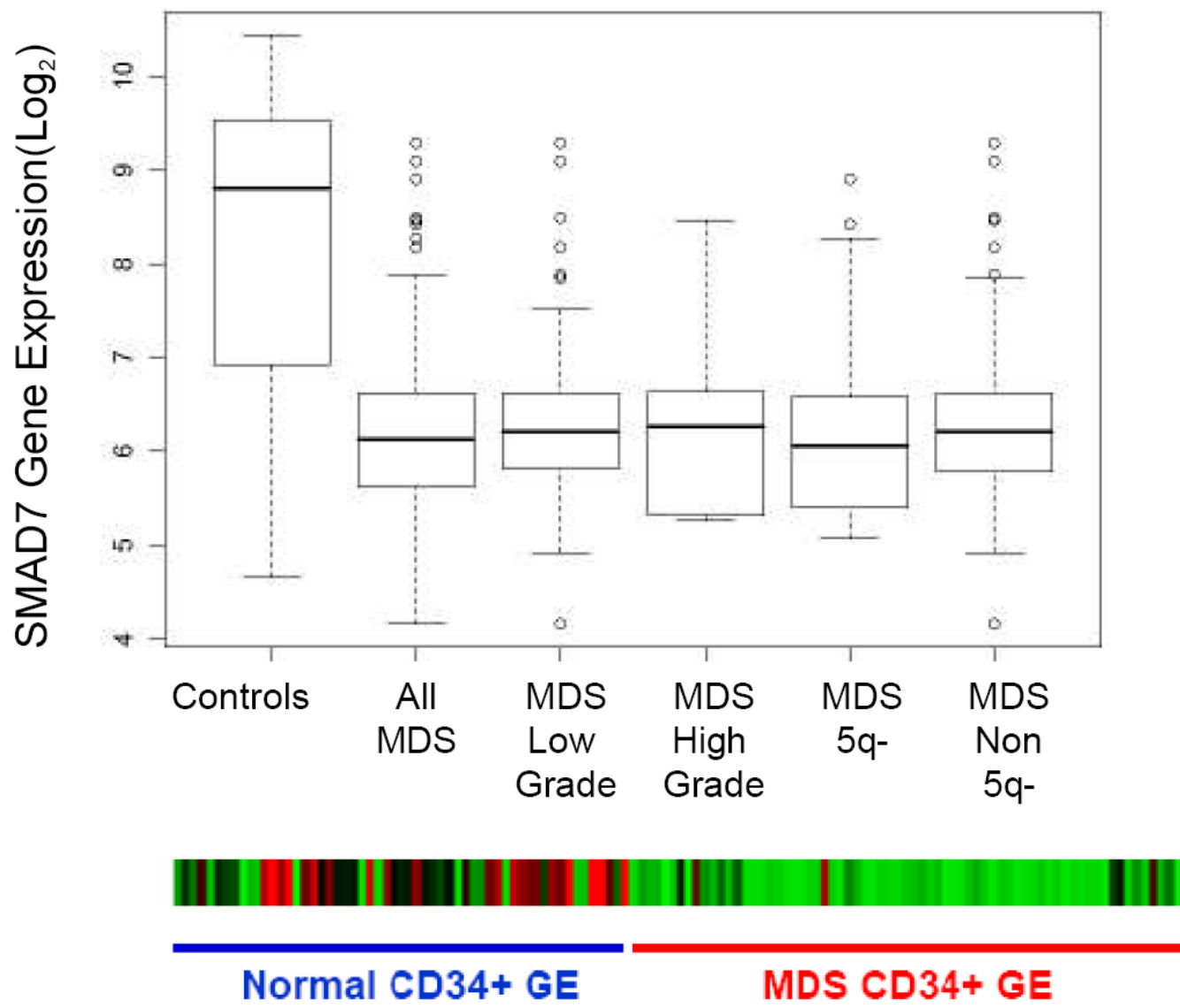

Color key

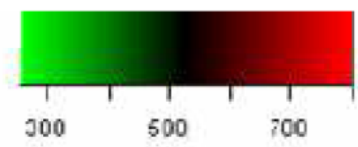

FIGURE 1. SMAD7 is significantly decreased in MDS

Differences in SMAD7 expression were also evaluated in normalized meta-analysis of MDS CD34+ (89 cases) and normal CD34+ (61 cases) cell derived gene expression microarray studies. Box plots show SMAD7 gene expression was significantly downregulated in MDS bone marrow CD34+ cells after multiple testing with Benjamin Hochberg correction with $\mathrm{p}$ value $<0.01$. The reduction is seen in both low $(n=83)$ and high grade $(n=6)$ cases and also in cases with $(n=29)$ and without $(n=60) 5 q$ deletion. Heat map shows individual values of all samples. 

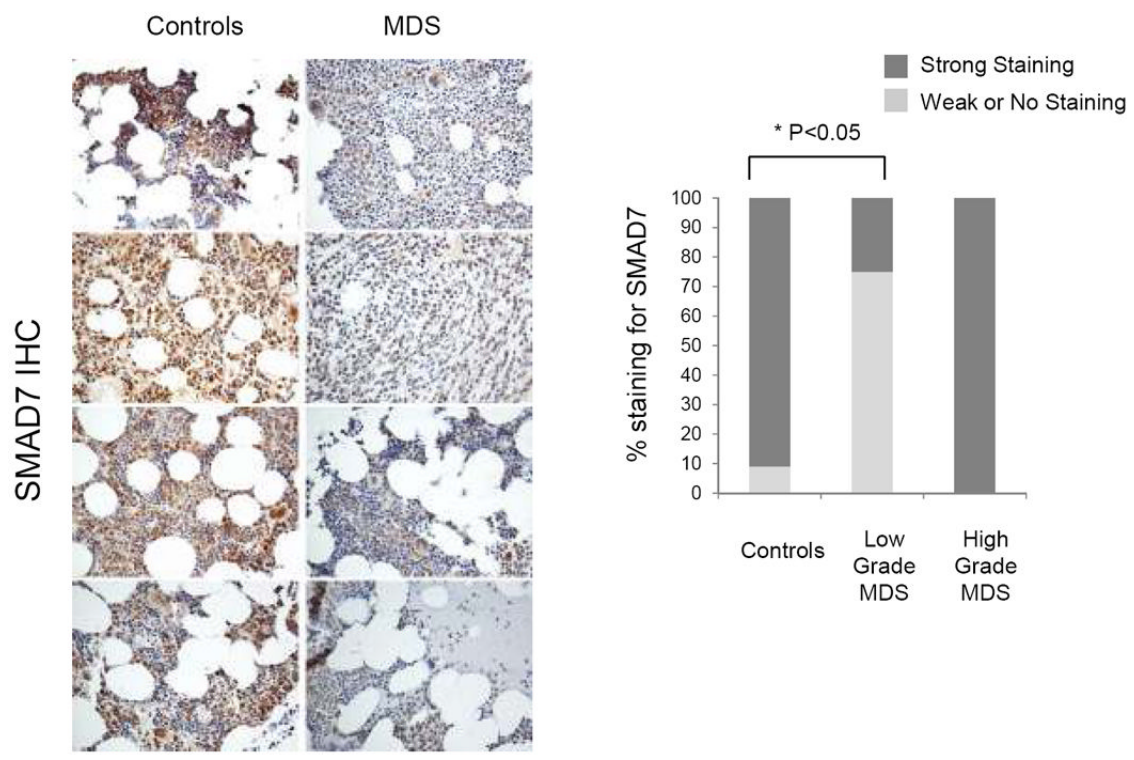

Figure 2. SMAD7 protein is decreased in MDS marrow progenitors

Tissue microarray was prepared from bone marrow (BM) biopsies from patients with MDS and controls with non-MDS Bone marrow biopsy samples were stained with SMAD7 antibody and show decreased expression in 4 representative cases of MDS when compared to controls. Low grade MDS samples revealed significantly less SMAD7 staining when compared to controls (Propotions test,p<0.05) 
A

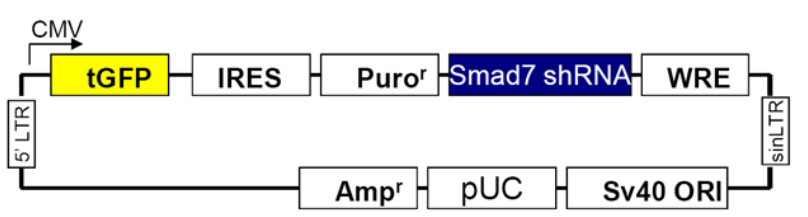

B

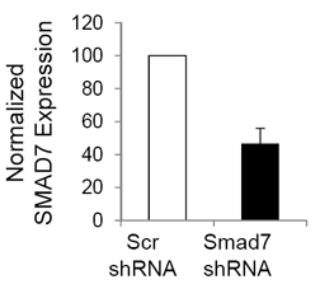

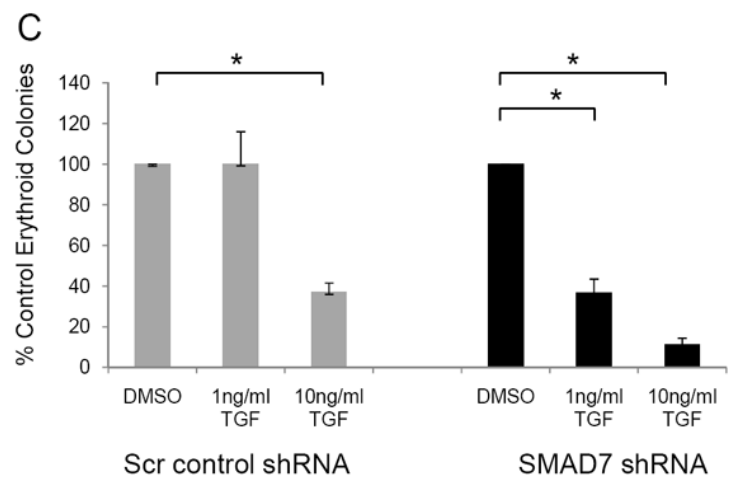

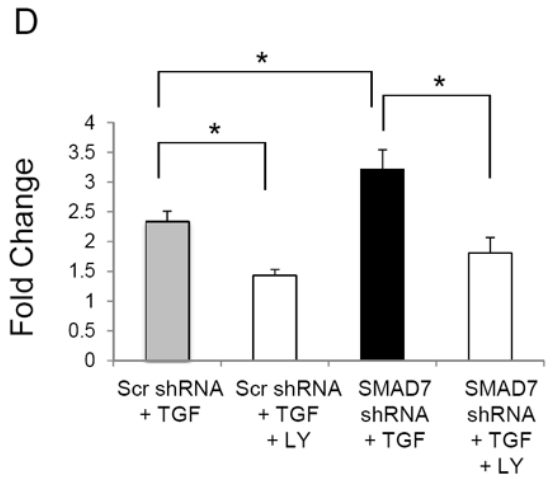

Figure 3. Reduction in SMAD7 can lead to increased sensitivity to suppressive effects of TGF- $\beta$ on hematopoiesis

GFP expressing Lentiviral based shRNA against SMAD7 (A) was used to knockdown SMAD7 in hematopoeitic cells. qPCR shows decrease in SMAD7 mRNA expression after lentiviral shRNA-TBRI infection in bone marrow stromal (HS-5) cells when compared to scrambled control (B). Primary CD34+ progenitors were electroporated with GFP coexpressing anti-SMAD7 shRNA construct and sorted after 48 hours. GFP positive cells were grown in methylcellulose with cytokines and erythroid colonies were counted after 14 days. SMAD7 shRNA transfected progenitors were significantly inhibited at lower concentrations of TGF-b when compared to cells transfected with scrambled control shRNA. Expressed as Means +/- s.e.m of 3 independent experiments. $(\mathrm{p}<0.05$, Two Tailed T test) (C). Bone marrow stroma derived cells (HS-5) with stable expression of anti-SMAD7 and control shRNAs were generated. These cells were transfected with plasmids expressing smad binding 3TP-Luciferase and beta-galactisidose (transfection control) and stimulated with TGF- $\beta 1$ in the presence and absence of LY-215 (dose .5uM). Tgf- $\beta 1$ induced control normalized Luciferase activity was significantly increased after SMAD7 knockdown. This increase was potently inhibited by LY-2157299.(D). 

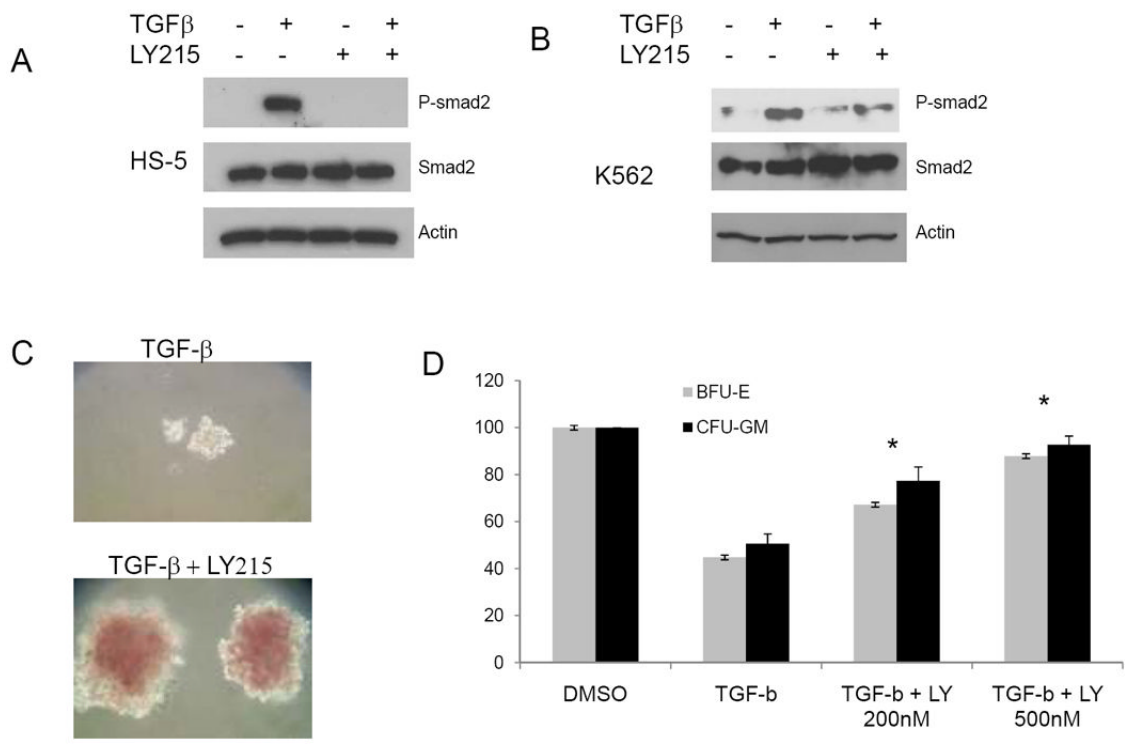

Figure 4. LY-2157299 is an effective and functionally active inhibitor of TGF- $\beta$ signaling in hematopoietic cells

Leukemic cells (K562) and marrow stromal cells (HS-5) were treated with TGF- $\beta 1$ (20ng/ $\mathrm{ml})$ in the presence and absence of LY-2157299 (.5uM) and assessed for SMAD2 phosphorylation by immunoblotting. LY-215 pretreament $(1 \mathrm{hr})$ led to attenuation of activation/phosphorylation of SMAD2 $(\mathbf{A}, \mathbf{B})$. Healthy CD34+ progenitors were plated in methylcellulose and cytokines in the presence and absence of TGF-b and TBRI inhibitor LY-2157299 (200nM and 500nM). Colonies were scored at Day 14 and results were expressed as means + S.E.M of 3 independent experiments. TGF-b exposure led to smaller (C) and lesser numbers (D) of colonies. These effects were reversed in the presence of LY215 treatment. 
A

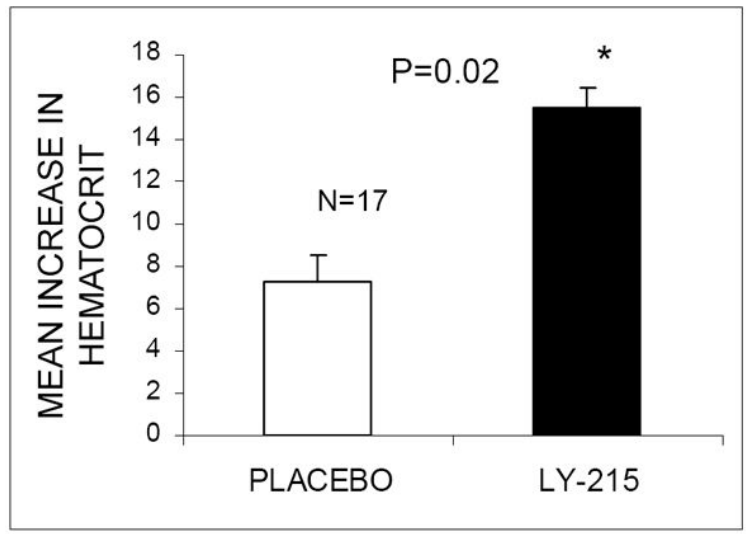

B Placebo

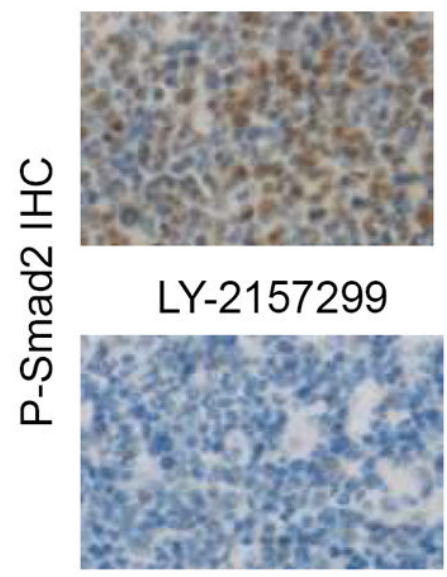

Figure 5. LY-2157299 can improve anemia in a murine model of TGF- $\beta 1$ driven bone marrow failure

Mice transgenic for alb/TGF- $\beta$ were treated with either LY-2157299 $(100 \mathrm{mg} / \mathrm{kg} / \mathrm{d})$ or vehicle (Placebo) daily by gastric lavage for 14 days. Blood counts were done on the $14^{\text {th }}$ day and revealed a significant rise in hematocrit after LY-2157299 treatment. (A) The mice were sacrificed and bone marrow biopsies were immunostained with antibody against phosphor-SMAD2. Representative sample shows inhibition of SMAD2 activation after LY-2157299 treatment. (B) 


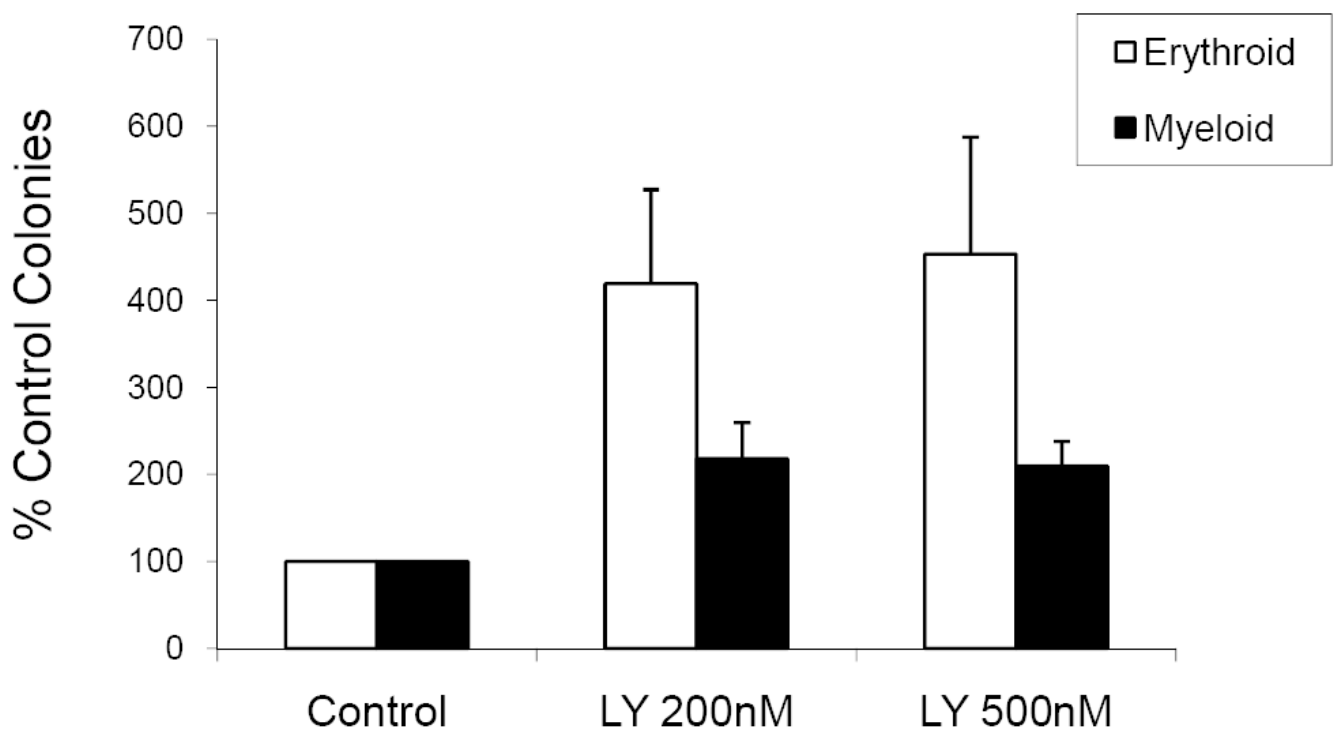

FIGURE 6. TBRI inhibition stimulates hematopoiesis in MDS

MDS Bone marrow derived MNCs from 10 patients were plated in methylcellulose and cytokines in the presence and absence of TBRI inhibitor LY-2157299 (200nM and 500nM). Colonies were scored at Day 14 and results were expressed as means + S.E.M of 10 independent experiments. 


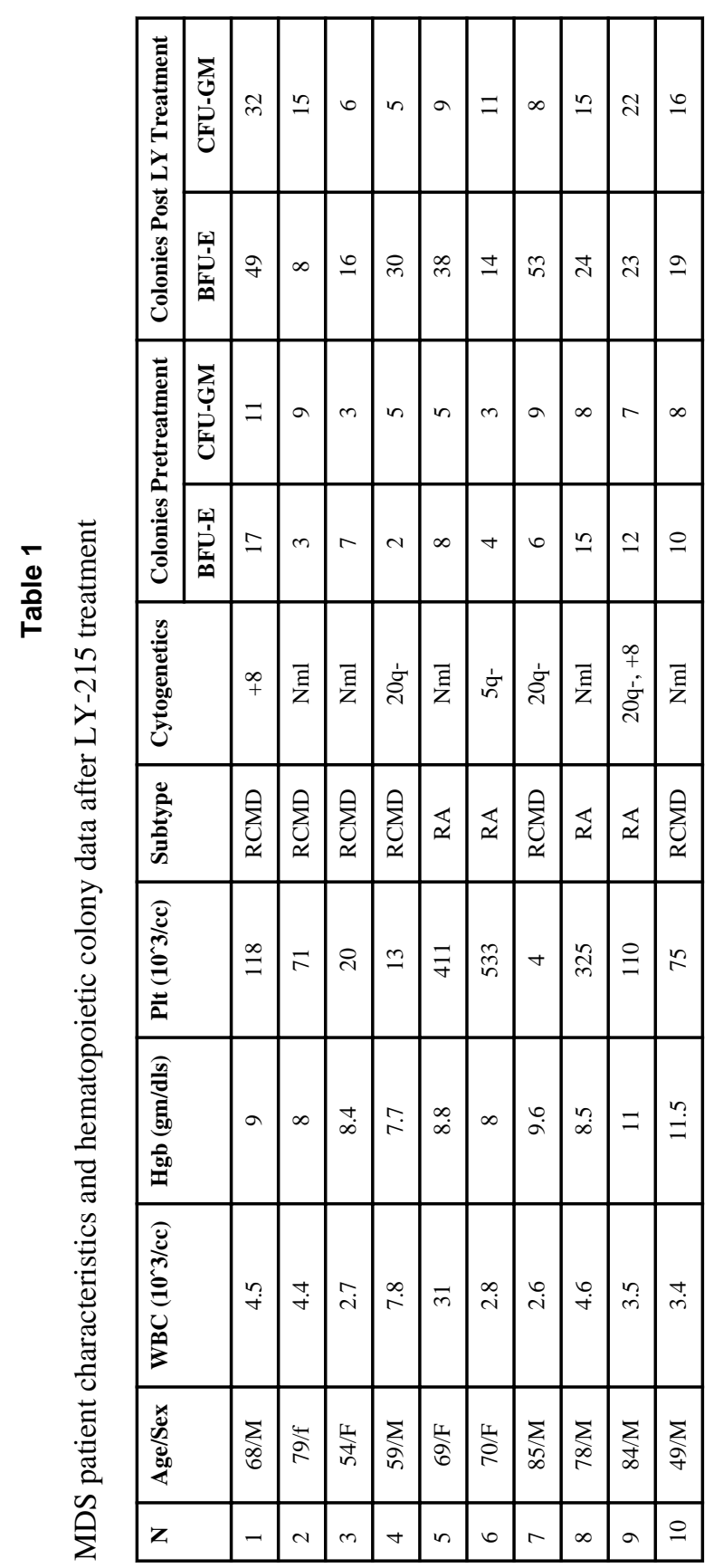

Cancer Res. Author manuscript; available in PMC 2012 February 1. 